\title{
TRADUCTION DE LA PARTICULE DE MISE EN RELIEF SLOVÈNE TUDI EN FRANÇAIS: CAS DE DISPARITIONS SURPRENANTES ${ }^{1}$
}

\section{INTRODUCTION}

Cet article se propose d'analyser tout d'abord le lexème slovène tudi (dont l'équivalent français le plus fréquent, dans un corpus comprenant quelque 300 exemples, est l'adverbe de phrase aussi), de passer ensuite en revue les cas de disparitions de ce dernier dans la traduction française, pour enfin conclure avec une analyse contrastive quant au fonctionnement du lexème slovène et de son équivalent français aussi, qui expliquera en grande mesure les cas de « disparitions ». La présente contribution montrera en effet s'il s'agit d'un phénomène aléatoire ou lié aux caractéristiques et contraintes de la langue française.

\section{DESCRIPTION FONCTIONNELLE ET CARACTÉRISTIQUES DU LEXÈME SLOVÈNE TUDI}

C'est en 1974 que le linguiste slovène J. Toporišič propose, dans un article intitulé Kratko oblikoslovje slovenskega jezika (Bref aperçu morphologique de la langue slovène), de remanier la classe hétérogène des adverbes slovènes (qui jusqu'alors regroupait aussi bien les particules que les adverbes) en proposant une délimitation formelle entre les particules et les adverbes, ajoutant aux classes de mots déjà existantes celle des particules. Depuis, la linguistique slovène distingue strictement la classe des adverbes et celle des particules (Černelič 1991 ; Smolej 2001).

Un lexème est qualifié d'adverbe (prislov) quand il peut former un constituant de la phrase (avec par conséquent un pronom interrogatif approprié) ; dans le cas contraire, il est rangé dans la classe des particules (členek) - (Toporišič 1974 ; Smolej 2001).

Le lexème tudi fait partie de la classe des particules, car ne pouvant pas à lui-seul assumer le rôle de constituant de phrase :

Jem tudi veliko sadja.

(Je mange aussi beaucoup de fruits.)

\footnotetext{
* Adresse de l'auteur : Filozofska fakulteta, Oddelek za romanske jezike in književnosti, Aškerčeva 2, 1000 Ljubljana, Slovénie. Mél : jacqueline.oven@guest.arnes.si

${ }^{1}$ La présente contribution résume une partie de la thèse de troisième cycle Skladenjsko vedenje francoskih ustreznikov slovenskega členka tudi, soutenue le 18 novembre 1999 à la Faculté des Lettres de Ljubljana (jury: prof. J. Orešnik - président, prof. V. Pogačnik - directeur de thèse, prof. B. Pogorelec-membre).
} 
Kaj ješ?

(Que manges-tu ?)

Tudi veliko sadje.

(Aussi beaucoup de fruits/ Beaucoup de fruits aussi.)

* Tudi.

* Aussi.

En nous référant aux travaux du linguiste danois H. Nølke (1990, 1993, 2001) sur les adverbes/adverbiaux de phrase en français, et plus particulièrement ceux portant sur les adverbes paradigmatisants, il convient néanmoins de souligner que la mise en relief peut porter sur l'ensemble du constituant ou sur une partie du constituant, d'où la pertinence de la distinction entre portée et foyer, telle que proposée par Nølke. La portée renferme toujours le foyer, alors que le foyer ne se recoupe pas toujours avec la portée de la focalisation.

Dans les deux exemples proposés, la portée de tudi est incontestablement veliko sadja, celle de aussi 'beaucoup de fruits'. La détermination du foyer, elle, est fortement déterminée par le contexte gauche : pour tudi le foyer pourrait être aussi bien veliko sadja que sadje, pour aussi 'beaucoup de fruits' ou seulement des fruits. Si on imagine un contexte gauche de type Jem veliko zelenjave (Je mange beaucoup de légumes), le foyer serait sadje (des fruits), alors que si on avait Jem zelenjavo (Je mange des légumes) le foyer serait veliko sadja (beaucoup de fruits).

La remarque précédente quant au renvoi nécessaire au contexte gauche permet de dégager la dimension contextuelle de la particule de mise en relief tudi et de déterminer une deuxième fonction la caractérisant, à savoir son rôle de connecteur (Toporišič 1976 ; Schlamberger Brezar 2000 ; Skubic 1999 ; Smolej 2001, 2007 ; Oven 1999, 2004). Dans le cadre de la théorie argumentative proposée par O. Ducrot (1980, 1980a, 1984), les concepts du posé et du présupposé permettent de mettre clairement en évidence la fonction de connecteur assumée par le lexème tudi, car renvoyant nécessairement au contexte gauche :

Jem tudi zelenjavo

(Je mange aussi des légumes)

Posé : Jem zelenjavo

(Je mange des légumes)

Présupposé: Jem druge stvari kot zelenjavo

(Je mange d'autres choses que des légumes)

Ces deux fonctions montrent le caractère particulier non seulement de cette particule qui assure à la fois un rôle intraphrastique (par le biais de la fonction de mise en relief) et interphrastique (par le biais de la fonction de connexion), mais aussi des particules de mise en relief slovènes en général (ce qui les démarque aussi bien des autres particules de non-mise en relief que d'ailleurs des conjonctions de coordination). Sa contribution quant au critère de cohérence est, en effet, double (Oven 1999, 2004) : tudi constitue en effet un élément anaphorique (de par sa fonction de conne- 
xion) et cataphorique (de par sa fonction de mise en relief). Ces constatations sont également vraies quant au fonctionnement du lexème français aussi.

Sur le plan sémantique, il faut souligner que le sème minimal de la particule tudi (et celle de aussi) est l'addition (qui découle de sa fonction de connecteur). L'emploi de tudi implique toujours un in (fr. et), ce qui est d'ailleurs également valable pour l'équivalent français aussi (A.-M. Perrin-Naffackh qualifie aussi d'adjonctif):

\author{
L'énoncé suivant \\ Jem tudi veliko zelenjave (Je mange aussi beaucoup de légumes) \\ implique \\ Jem nekaj (kar ni zelenjava) IN veliko zelenjave \\ (Je mange quelque chose - qui n'est pas un légume - ET beaucoup de légumes).
}

Nous pouvons donc en conclure que les deux fonctions inhérentes au lexème slovène tudi et à son équivalent français le plus fréquent aussi sont la mise en relief (intraphrastique) et la connexion (interphrastique). Cette constation aura toute son importance lors de l'analyse des cas de disparition du lexème slovène tudi dans la traduction française.

\title{
2. CAS DE DISPARITIONS
}

Les cas de disparitions ont été relevés dans un corpus constitué à partir d'exemples recueillis dans le roman slovène Alamut de V. Bartol, traduit en français par C. Vincenot. Le corpus comprend quelque 300 exemples. Dans environ un quart des exemples, force est de constater que le lexème slovène tudi se perd dans la traduction française.

Passons en revue quelques exemples pour ensuite en dégager une conclusion éventuelle quant aux différences de fonctionnement des particules de mise en relief slovènes et françaises. En observant ces exemples de plus près, nous constatons qu'ils présentent tous le même cas de figure : l'une des deux fonctions inhérentes au lexème tudi (mise en relief et connexion) est déjà présente dans le contexte immédiat. Dans ces cas de figure, on observe alors une non-traduction de tudi.

Nous allons nous focaliser sur les exemples présentant déjà une idée d'addition (au sens large du terme) dans le contexte immédiat où apparait le lexème slovène tudi (dont l'addition est le sème minimal découlant de sa fonction de connexion).

L'addition, outre celle inhérente au lexème tudi, est exprimée aussi bien par le biais de mots grammaticaux que de mots sémantiquement pleins. Ainsi trouvonsnous parmi les mots grammaticaux des prépositions (poleg ... tudi/ outre ; poleg ... tudi/ en plus de) et des conjonctions (in ... tudi/ et). Les lexèmes sémantiquement pleins se présentent sous forme d'adverbe (končno ... tudi/enfin), de verbe (biti med ... tudi/ être parmi ; biti med ... tudi/faire partie de ; prenesti se tudi na/ se communiquer à ; združiti tudi/ associer à) ou d'adjectif (tudi drugi/ autres ; tudi ostali/ autres ; tudi vsi/ tous ; tudi zadnji/ dernier ; tudi poslednji/dernier).

Nous réintroduirons le lexème français aussi dans tous les exemples présentant un cas de disparition du lexème slovène tudi dans la traduction française pour établir une évaluation entre les exemples sans et avec aussi. 
poleg ... tudi : outre

Dai Ibrahim je poleg dogmatike in arabske slovnice razlagal tudi koran ter učil algebro in ostale računske nauke. (p. 122)

Outre la dogmatique et la grammaire arabe, le dey Ibrahim leur enseignait le Coran, l'algèbre et les autres sciences du calcul. (p. 118)

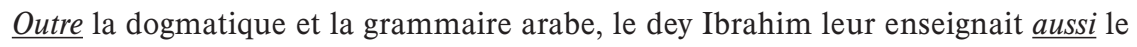
Coran, l'algèbre et les autres sciences du calcul.

poleg ...tudi : en plus de

Poleg vojaških veščin bi se morali učiti tudi dogmatike in zgodovine izmailstva. (p. 392)

En plus des sciences militaires, les hommes devaient étudier la dogmatique et l'histoire de l'ismaïlisme. (p. 388)

En plus des sciences militaires, les hommes devaient aussi étudier la dogmatique et l'histoire de l'ismailisme.

in ... tudi : et

Tam sem bil rojen in sem preživel tudi svojo mladost. (p. 503)

Ma famille est originaire de Ghazna. J'y suis né et j’y ai passé ma jeunesse. (p. 497)

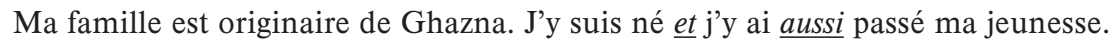

končno ... tudi : enfin

Veliki dai je obhodil čete, da se prepriča o njihovi pripravljenosti. Vojak je stopal za njim in vodil njegovega konja za vajeti. Končno sta prišla tudi $k$ fedaijem in Abu Ali je zlezal na vrh stolpa. (p. 227)

Le grand dey fit le tour du détachement afin de s'assurer que chacun était à son poste. Un soldat marchait derrière lui, tenant son cheval par la bride. Ils s'approchèrent enfin des fédayens. (p. 223)

* Le grand dey fit le tour du détachement afin de s'assurer que chacun était à son poste.Un soldat marchait derrière lui, tenant son cheval par la bride. Ils s'approchèrent aussi enfin des fédayens.

biti med ... tudi : être parmi, faire partie de

$V$ Kabulu sem bil nekoč med mnogimi drugimi tudi jaz gost bogatega indijskega kneza. (p. 259)

A Kaboul, il y a de cela longtemps, j'ai été un jour, moi parmi d'autres, l'hôte d'un riche prince natif de l'Inde. (p. 256)

A Kaboul, il y a de cela longtemps, j'ai été un jour, moi aussi parmi d'autres, l'hôte d'un riche prince natif de l'Inde. 
Ta mu je pomagal, da je zbral okrog sebe četico sedemdesetih mož, $\underline{\text { med katerimi je bil tudi }}$ podčastnik, ki mi je pripovedoval to zgodbo. (p. 140)

Celui-ci l'aida à rassembler une petite compagnie de dix hommes, dont faisait partie le sousofficier qui m'a conté toute l'histoire. (p. 136)

Celui-ci l'aida à rassembler une petite compagnie de dix hommes, dont faisait aussi partie le sous-officier qui m'a conté toute l'histoire.

\section{prenesti se tudi na : se communiquer à}

Nemir, ki se je polastil moštva, se je prenesel tudi na živali. Zganile so se in postale nestrpne. (p. 443)

L'impatience de la troupe se communiquait aux bêtes, qui manifestèrent quelques signes de nervosité. (p. 438)

L'impatience de la troupe se communiquait aussi aux bêtes, qui manifestèrent quelques signes de nervosité.

\section{združiti tudi : associer à}

S pesništvom so bile združene tudi vaje v govorništvu. (p. 127)

A la métrique étaient associés des exercices de rhétorique. (p. 124)

A la métrique étaient aussi associés des exercices de rhétorique.

\section{tudi drugi : les autres}

Halima je takoj spoznala svojo lastnino. Toliko da se ni od strahu sesedla. Nobene besede ni mogla spraviti iz sebe. Tudi druge so se prestrašile. (p. 382)

Halima reconnut tout de suite son bien et manqua s'évanouir de frayeur. Elle était incapable d'articuler le moindre mot. Les autres cependant n'avaient pas l'air mieux à leur aise. (p. 377) Halima reconnut tout de suite son bien et manqua s'évanouir de frayeur. Elle était incapable d'articuler le moindre mot. Les autres non plus cependant n'avaient pas l'air mieux à leur aise.

\section{tudi ostali : les autres}

Poveljnik oddelka je pridržal konja in velel tudi ostalim, naj postoje. (p. 54)

Le chef du détachement retint son cheval et commanda aux autres de s'arrêter. (p. 52)

Le chef du détachement retint son cheval et commanda aussi aux autres de s'arrêter.

tudi vsi : tous

Trije odposlanci, pa tudi vsi, ki so ostali v bližini, so odreveneli od groze. (p. 447)

Les rois envoyés et tous ceux qui assistaient à la scène étaient pétrifiés d'effroi. (p. 442)

Les rois envoyés et tous ceux aussi qui assistaient à la scène étaient pétrifiés d'effroi. 


\section{tudi zadnji/poslednji : dernier}

Vsi so potrdili krivdo. Pri vsakem imenu se je Hosein zdrznil. Ves čas je na tihem upal, da se bo nekdo uprl, da bo nekdo uvidel, da je bil on v pravici in da ni mogel ravnati drugače. Ko je tudi poslednji izrekel svoj " kriv », je zatulil: "Zločinski psi ». (p. 517)

Le verdict était acquis à l'unanimité. A chaque nom, Hossein avait réprimé un tressaillement. Jusqu'au bout il avait espéré en secret que quelqu'un s'opposerait à la sentence, qu'une voix s'élèverait pour rappeler qu'il était dans son droit, qu'il n'avait agi que pour défendre l'honneur de son rang. Lorsque tomba la dernière sentence, il leur hurla à la face : "Chiens criminels ». (p. 511-512)

* Le verdict était acquis à l'unanimité. A chaque nom, Hossein avait réprimé un tressaillement. Jusqu'au bout il avait espéré en secret que quelqu'un s'opposerait à la sentence, qu'une voix s'élèverait pour rappeler qu'il était dans son droit, qu'il n'avait agi que pour défendre l'honneur de son rang. Lorsque tomba aussi la dernière sentence, il leur hurla à la face : "Chiens criminels".

Dans ce dernier cas, il serait néanmoins possible d'introduire enfin à la place de aussi :

Le verdict était acquis à l'unanimité. A chaque nom, Hossein avait réprimé un tressaillement. Jusqu'au bout il avait espéré en secret que quelqu'un s'opposerait à la sentence, qu'une voix s'élèverait pour rappeler qu'il était dans son droit, qu'il n'avait agi que pour défendre l'honneur de son rang. Lorsque tomba enfin la dernière sentence, il leur hurla à la face : " Chiens criminels ».

Dans la majorité des cas, l'ajout de aussi serait possible, la phrase n'en serait pas pour autant agrammaticale, néanmoins il serait ressenti comme redondant. Le slovène, lui, maintient les deux moyens d'expression de l'addition, d'où deux interprétations possibles : soit le lexème slovène se vide de sa fonction de connecteur au profit d'un autre lexème, soit il participe au renforcement de la cohésion textuelle (puisque deux éléments assurent la progression thématique). Néanmoins l'introduction de aussi n'est pas possible quand il y a expression d'une forme particulière de l'addition, à savoir l'élément terminal d'une liste ou l'aboutissement d'un processus.

La non-traduction en français porte toujours sur la particule de mise en relief tudi, c'est à dire que le français préserve l'autre moyen exprimant l'addition au détriment de tudi. La question qui se pose est de savoir si la non-traduction de tudi en français n'entraîne pas un affaiblissement de la fonction de mise en relief inhérente à tudi. Cet affaiblissement semble être le plus marqué dans le cas d'équivalents français sémantiquement pleins. La fonction de connecteur, elle, est préservée et assurée en français par le biais de l'autre lexème traduisant l'addition.

Dans les exemples suivants, nous constatons également que l'addition peut prendre dans certains cas une valeur sémantique particulière, à savoir l'identité, qui est elle aussi rendue par le biais de mots grammaticaux (kakor ... tudi/ comme ; tudi ... 
obenem/ en même temps) que de mots sémantiquement pleins (tudi isti/ même ; tudi ... podoben - pareil/ en tout point semblable); tudi ... slediti/ suivre son exemple ; tudi... nič drugače od/ se ressembler comme).

Nous réintroduirons également aussi dans les exemples choisis pour en dégager d'éventuelles caractéristiques quant au fonctionnement des deux langues.

kakor... tudi : comme/tout comme

Kakor Mirjam, je bila tudi Širin kristjanka. (p. 97)

Comme Myriam, Shirin était d'origine chrétienne. (p. 94)

Comme Myriam, Shirin était aussi d'origine chrétienne.

In prav kakor pred pol leta je prišel tudi v začetku zime reis Abal Fazel Lumbani iz Raija z važnim sporočilom na grad. (p. 536)

Au début de l'hiver, on vit arriver au château le raïs Abul Fazel Lumbani, de Raï. Tout comme six mois auparavant, il était porteur d'un message important. (p. 531)

Au début de l'hiver, on vit arriver au château aussi le raïs Abul Fazel Lumbani, de Raï.

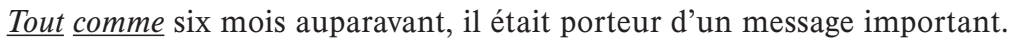

\section{tudi ...obenem : en même temps}

Zdelo se mi je, da nekoga pozdravlja in se mu klanja. Obenem z njim se je pripogibal tudi minaret. (p. 323)

Il me semblait ensuite qu'il répondait au salut de quelqu'un: il s'inclinait avec respect, et le minaret se courbait en même temps que lui. (p. 321)

Il me semblait ensuite qu'il répondait au salut de quelqu'un : il s'inclinait avec respect, et le minaret aussi se courbait en même temps que lui.

Sleherni vojak se je izuril v železnega vojaka in sleherni vojak je obenem tudi najbolj goreč vernik. (p. 145)

Chaque croyant est un soldat trempé comme l'acier. Et chaque soldat est en même temps le plus ardent des croyants. (p. 142)

Chaque croyant est un soldat trempé comme l'acier. Et chaque soldat est en même temps aussi le plus ardent des croyants.

tudi isti : même

Poznejši vezir je bil kakor jaz iz Tusa in tudi isto ime je imel kot jaz: Hasan ben Ali. (p. 189)

Le futur vezir était comme moi natif de Tus et portait le même nom que moi Hassan Ibn Ali.

(p. 186)

Le futur vezir était comme moi natif de Tus et portait aussi le même nom que moi :

Hassan Ibn Ali. 
tudi ...podoben : pareil / en tout point semblable

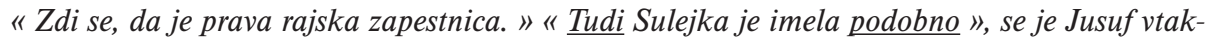
nil $v$ besedo. (p. 368)

" Cela semble bien être un véritable bracelet du paradis. " "Suleïka en avait un pareil ", intervint Yusuf. (p. 364)

"Cela semble bien être un véritable bracelet du paradis. " "Suleïka aussi en avait un pareil ", intervint Yusuf.

Emirju Arslanu Tašu je poslal ukaz, da najpozneje v enem mesecu osvoji in poruši Alamut /.../ Podobno povelje je izdal tudi Kizil Sariku, ki še zmerom zaman oblega trdnjavo Zur Gumbadan v Kuzistanu. (p. 403)

Il avait par ailleurs donné un mois à l'émir Arslan Tash pour s'emparer d'Alamut, avec ordre de raser la forteresse, faute de quoi il se verrait automatiquement accusé de haute trahison. Et une directive en tout point semblable avait été dépêchée au dénommé Kizil Sarik, qui campait toujours en vain sous les murs de la citadelle de Zur Gumbadan, dans le Kuzistan ... (p. 398) Il avait par ailleurs donné un mois à l'émir Arslan Tash pour s'emparer d'Alamut, avec ordre de raser la forteresse, faute de quoi il se verrait automatiquement accusé de haute trahison. Et une directive en tout point semblable avait aussi été dépêchée au dénommé Kizil Sarik, qui campait toujours en vain sous les murs de la citadelle de Zur Gumbadan, dans le Kuzistan ...

tudi ... slediti : suivre son exemple

Stopil je od nadzidkov in legel na blazine, ki so bile razprostrte na tleh. Pozval je tudi prijatelja, naj mu sledita. (p. 298)

Il s'éloigna du parapet, s'allongea sur les coussins disposés à même le sol et invita ses deux amis à suivre son exemple. (p. 296)

Il s'éloigna du parapet, s'allongea sur les coussins disposés à même le sol et invita aussi ses deux amis à suivre son exemple.

tudi ... biti nič drugače od : ressembler comme

"Opazil sem, da so vam vsem obrazi nekam ostri in trdi, kot da bi vam bilo trideset let. A po rasti brade bi vam jih prisodil komaj dvajset. /.../ " " Počakaj štirinajst dni in tudi ti ne boš nič drugačen od nas. " (p. 61)

"Tout à l'heure j'ai observé vos visages : tous sont durs et marqués; on vous donnerait facilement trente ans, pourtant l'on voit bien à vos barbes que la plupart d'entre vous en ont à peine vingt. /.../" "Attends seulement quinze jours et tu nous ressembleras comme un frère. " (p. 59) " Tout à l'heure j'ai observé vos visages : tous sont durs et marqués ; on vous donnerait facilement trente ans, pourtant l'on voit bien à vos barbes que la plupart d'entre vous en ont à peine vingt. /.../ " "Attends seulement quinze jours et tu nous ressembleras, toi aussi, comme un frère. " 
Les conclusions sont les mêmes que celles faites après avoir passé en revue les premiers exemples présentant une double expression de l'addition : l'ajout de aussi serait possible bien que redondant, sans pour autant affecter l'acceptabilité grammaticale de la phrase. Le français ne retient qu'un seul des deux moyens exprimant l'addition (privilégiant la «non-particule »), alors que le slovène, lui, maintient les deux moyens traduisant l'addition.

Il est intéressant de noter que l'emploi de certains équivalents potentiels de aussi (et plus particulièrement le lexème également) nous amène aux mêmes conclusions que celles énoncées pour le lexème aussi, à savoir une acceptabilité grammaticale avec, toutefois, un effet de redondance :

Dai Ibrahim je poleg dogmatike in arabske slovnice razlagal tudi koran ter učil algebro in ostale računske nauke. (p. 122)

Outre la dogmatique et la grammaire arabe, le dey Ibrahim leur enseignait le Coran, l'algèbre et les autres sciences du calcul. (p. 118)

Outre la dogmatique et la grammaire arabe, le dey Ibrahim leur enseignait aussi le Coran, l'algèbre et les autres sciences du calcul.

Outre la dogmatique et la grammaire arabe, le dey Ibrahim leur enseignait également le Coran, l'algèbre et les autres sciences du calcul.

Tam sem bil rojen in sem preživel tudi svojo mladost. (p. 503)

Ma famille est originaire de Ghazna. J'y suis né et j'y ai passé ma jeunesse. (p. 497)

Ma famille est originaire de Ghazna. J'y suis né et j'y ai aussi passé ma jeunesse.

Ma famille est originaire de Ghazna. J'y suis né et j'y ai également passé ma jeunesse.

Poveljnik oddelka je pridržal konja in velel tudi ostalim, naj postoje. (p. 54)

Le chef du détachement retint son cheval et commanda aux autres de s'arrêter. (p. 52)

Le chef du détachement retint son cheval et commanda aussi aux autres de s'arrêter.

Le chef du détachement retint son cheval et commanda également aux autres de s'arrêter.

Kakor Mirjam, je bila tudi Širin kristjanka. (p. 97)

Comme Myriam, Shirin était d'origine chrétienne. (p. 94)

Comme Myriam, Shirin était aussi d'origine chrétienne.

Comme Myriam, Shirin était également d'origine chrétienne.

Il conviendrait néanmoins de vérifier si ces conclusions s'appliquent aux autres équivalents potentiels de aussi (encore, par ailleurs, ...) et si leurs emplois ne seraient pas soumis à un certain nombre de conditions.

Terminons par un exemple illustrant un aspect contrastif révélateur quant au fonctionnement des deux langues face au phénomène de la redondance :

Užival je neomejeno zaupanje vladarja, vse dokler ni zašel z mlado sultanjo v spor zaradi prestolonasledstva. Tudi prej so ga že bili zavistneži in nasprotniki pri cesarju črnili. Toda sultan jih ni poslušal. (p. 419) 
Ainsi avait-il joui longtemps de la confiance illimitée du souverain /.../ jusqu'à ce qu'il entrât en conflit avec la jeune sultane au sujet de la succession du trône. Bien des fois auparavant, envieux et adversaires de tout poil l'avaient noirci aux yeux du sultan, mais celui-ci ne les écoutait pas... (p. 415)

* Ainsi avait-il joui longtemps de la confiance illimitée du souverain /.../ jusqu'à ce qu'il entrât en conflit avec la jeune sultane au sujet de la succession du trône. Bien des fois *aussi auparavant, envieux et adversaires de tout poil l'avaient noirci aux yeux du sultan, mais celui-ci ne les écoutait pas...

Nous constatons que la réintroduction de aussi entraîne bien plus qu'un effet de redondance, rendant la phrase même agrammaticale. Les deux éléments contribuant à l'expression de l'addition en slovène že et tudi sont en effet tous deux des particules de mise en relief. Le slovène permet leur cohabitation, alors qu'en français cette combinatoire est impossible, car menant à des phrases grammaticalement inacceptables. Nous sommes donc en face d'un exemple de redondance, mais cette fois-ci il s'agit d'une redondance fonctionnelle et non purement sémantique. Cet exemple révèle un fonctionnement différent dans les deux langues, phénomène (redondance fonctionnelle) qu'il serait d'ailleurs intéressant d'observer sur d'autres particules de mise en relief ou segments de la langue et qui permettrait éventuellement de dresser un tableau contrastif plus global entre le slovène et le français.

\section{CONCLUSION}

En conclusion nous pouvons constater que les cas de non-traduction du lexème slovène tudi en français se produisent quand le contexte immédiat renferme un autre élément exprimant déjà l'addition, au sens large du terme. Les moyens additifs sont extrêmement variés dans les deux langues, incluant aussi bien des mots grammaticaux que des lexèmes sémantiquement pleins. Nous avons également constaté que la réintroduction du lexème français aussi était, dans la plupart des cas de figure, possible bien que redondante, la phrase restant grammaticalement correcte. Nous avons relevé toutefois deux cas de figure ne permettant pas la réintroduction de aussi, à savoir un exemple particulier d'addition (l'élément terminal d'une liste ou l'aboutissement d'un processus), ainsi que la cohabitation de deux particules de mise en relief en français, alors que le slovène permet ce genre de combinatoire. L'emploi de aussi est ainsi justifié s'il remplit ses deux fonctions : celle de mise en relief et celle de connecteur, alors que le slovène peut employer tudi même si l'une de ces deux fonctions est déjà assurée dans le contexte immédiat. En shématisant, nous pouvons donc constater que le lexème slovène tudi est un moyen de mise en relief et/ou connecteur, alors que le lexème français aussi est un moyen de mise en relief et connecteur. Ces observations permettent d'émettre des hypothèses plus générales quant au fonctionnement des deux langues. Le français semble, en effet, beaucoup plus réticent au phénomène de la redondance, le slovène, lui, y est beaucoup plus enclin, peut-être parce que ne la percevant pas comme telle, mais plutôt comme un 
moyen de cohésion renforcée. Il serait intéressant d'analyser le fonctionnement des autres particules de mise en relief en slovène et en français qui confirmerait ou réfuterait les constatations faites dans la présente contribution.

Le rapport des deux langues au phénomène de la redondance pourrait faire, lui aussi, l'objet d'une étude plus approfondie, incluant d'autres domaines que celui des particules de mise en relief.

\section{Corpus}

BARTOL, Vladimir (1988) Alamut. Ljubljana : Mladinska knjiga.

BARTOL, Vladimir (1988) Alamut. Traduit par Claude Vincenot, adapté par J.-P. Sicre. Paris : Phébus.

\section{Bibliographie}

ČERNELLČ, Ivanka (1991) « Členek kot besedna vrsta v slovenskem knjižnem jeziku. » Jezikoslovni zapiski 1, 73-85.

ČERNELIČ-KoZLEVČAR, Ivanka (1993) «O delitvi členkov. » In : Vprašanja slovarja in zdomske književnosti/Zborovanje slavistov, Murska Sobota 1992. Ljubljana : Zavod RS za šolstvo in šport, 213-227. (Zbornik Slavističnega društva Slovenije, 3).

Ducrot, Oswald (1980) Les échelles argumentatives. Paris : Editions de Minuit.

Ducrot, Oswald (1980 a) Les mots du discours. Paris : Editions de Minuit.

Ducrot, Oswald (1984) Le dire et le dit. Paris : Editions de Minuit.

Langue Française (1990), no. 88, Classification des adverbes.

NøLKe, Henning (1983) «Problems in the semantic/pragmatic description of French adverbials like même, aussi, surtout and seulement. » Acta Linguistica Hafniensia 17/2, 59-71.

NøLKE, Henning (1990) «Classification des adverbes. » Langue française 88, 3-4, 12-27.

NøLKE, Henning (1993) Le regard du locuteur. Paris : Kimé.

NøLKE, Henning (2001) Le regard du locuteur 2. Paris : Kimé.

OVEN, Jacqueline (1999) Skladenjsko vedenje francoskih ustreznikov slovenskega členka tudi. Magistrsko delo. [Thèse de 3ème cycle]. Ljubljana : Univerza v Ljubljani, Filozofska fakulteta.

Oven, Jacqueline (2004) « Le lexème slovène aussi et ses équivalents français.» Linguistica, 75-88.

Perrin-NAFFaKH, A.-M. (1996) « Aussi adjonctif : de la syntaxe à la sémantique. » Le Français moderne LXIV/2, 136-155.

SChLAmberger BREZAR, Mojca (2000) « Les connecteurs en combinaison avec les marqueurs modaux : l'exemple du français et du slovène. » Linguistica XL/2, 273-282.

SKUBIC, Andrej (1999) « Ogled kohezijske vloge slovenskega členka. » Slavistična revija 47/2, 211-238.

SMolej, Mojca (2001) « Členek v slovenskem knjižnem jeziku. Pomenoslovni in skladenjski vidiki. » Ljubljana : magistrsko delo.

SMolej, Mojca (2004) « Členki kot besedilni povezovalci. » Jezik in slovstvo 49/5, 45-57.

SMoleJ, Mojca (2007) « La classification des particules en français et en slovène » Lingusitica $47,109-120$.

TopoRIšlČ, Jože (1974) « Kratko oblikoslovje slovenskega jezika. » In : M. Kmecl et al. (dir.), Slovenski jezik, literatura in kultura. Ljubljana : Seminar slovenskega jezika, literature in kulture pri Oddelku za slovanske jezike in književnosti Filozofske fakultete Univerze, 29-50.

TopoRIššč, Jože (1976) Slovenska slovnica. Maribor : Založba Obzorja Maribor. 


\section{Résumé \\ TRADUCTION DE LA PARTICULE DE MISE EN RELIEF SLOVÈNE TUDI EN FRANÇAIS : CAS DE DISPARTIONS SURPRENANTES}

La présente contribution se propose d'analyser les cas de disparitions de la particule de mise en relief slovène tudi dans les traductions françaises. Dans le corpus choisi (le roman Alamut de V. Bartol et sa traduction française), nous avons constaté la non-traduction de la particule slovène dans presque un tiers des cas. Le lexème tudi est caractérisé par deux fonctions : la mise en relief et la connexion. Sur le plan sémantique, il renferme un sème minimal, à savoir l'addition. L'analyse des exemples présentant des cas de non-traduction de la particule slovène tudi en français a abouti aux conclusions suivantes: il y a non-traduction quand le contexte immédiat inclut un autre élément exprimant déjà l'addition. La réintroduction du lexème aussi (l'équivalent le plus fréquent de la particule tudi) s'avère être redondante, sans pour autant, dans la grande majorité des cas, affecter l'acceptabilité, voire la grammaticalité de la phrase. Le rajout du lexème aussi rend la phrase agrammaticale dans seulement deux cas de figure : quand l'addition exprime l'élément terminal d'une liste et quand le contexte minimal renferme déjà une autre particule de mise en relief (sl. tudi že - fr. * aussi déjà). D'un point de vue contrastif, nous constatons que tudi et aussi assument les deux mêmes fonctions (mise en relief et connexion) dans les deux langues, avec néanmoins une différence majeure au niveau de la combinatoire : alors que la particule slovène tudi assure la mise en relief et/ou la connexion, le lexème aussi, lui, ne peut être que moyen de mise en relief et de connexion. Le français évite toute forme de redondance sémantique, tout en excluant la redondance fonctionnelle, alors que le slovène permet ces cas de figure.

\section{Povzetek \\ PREVAJANJE SLOVENSKEGA ČLENKA TUDI V FRANCOŠČINO: PRESENETLJIVA IZGINOTJA V FRANCOSKEM PREVODU}

Avtorica $\mathrm{v}$ članku obravnava slovenski členek tudi in njegov izpad $\mathrm{v}$ francoskem prevodu. $\mathrm{V}$ izbranem korpusu (Bartolov roman Alamut v slovenskem izvirniku in francoski različici) členek izpade skoraj v četrtini primerov. Členek tudi uvrščamo med poudarjalne členke z besediloslovno navezovalno vlogo. $\mathrm{S}$ funkcijskega vidika je torej poudarjalen in navezovalen, pri čemer je njegova minimalna pomenska prvina dodajalnost. Preučitev primerov, kjer pride do izpada oziroma do neprevajanja slovenskega členka tudi $v$ francoskem prevodu, je pripeljala do naslednjega zaključka: vsi primeri vsebujejo v ožjem sobesedilu že izraženo dodajalnost z drugimi sredstvi. V takšnih primerih pride do izpada slovenskega tudi v francoskem prevodu. Vnos leksema aussi (sicer najbolj pogostega ustreznika členka tudi) je redundanten, pri čemer ostaja poved jezikovno večinoma sprejemljiva. Vnos leksema aussi je jezikovno nesprejemljiv v primeru, da dodajalnost izraža končnost ali da imamo v ožjem sobesedilu že en poudarjalni členek ( ${ }^{*} t u d i \check{z} e$ ). Če torej primerjamo tudi in aussi, ki funkcijsko sovpadata, je njuna glavna razlika $\mathrm{v}$ tem, da je slovenski členek poudarjalen in/ali navezovalen, medtem ko je francoski aussi poudarjalen in navezovalen. Francoščina je torej vsekakor manj naklonjena redundanci kot slovenščina (dopušča sicer semantično redundanco, čeprav se ji močno izogiba, medtem ko funkcijsko redundanco strogo odklanja). 